Jean Alric, Xenie Johnson

CEA, CNRS, Aix-Marseille Université, Institut de Biosciences et Biotechnologies Aix-Marseille, UMR 7265, Laboratoire de Bioénergétique et Biotechnologie des Bactéries et Microalgues, CEA Cadarache, Saint-Paul-lez-Durance F-13108, France

Photosynthetic reactions proceed along a linear electron transfer chain linking water oxidation at photosystem II (PSII) to $\mathrm{CO}_{2}$ reduction in the Calvin-Benson-Bassham cycle. Alternative pathways poise the electron carriers along the chain in response to changing light, temperature and $\mathrm{CO}_{2}$ inputs, under prolonged hydration stress and during development. We describe recent literature that reports the physiological functions of new molecular players. Such highlights include the flavodiiron proteins and their important role in the green lineage. The parsing of the proton-motive force between $\Delta \mathrm{pH}$ and $\Delta \psi$, regulated in many different ways (cyclic electron flow, ATPsynthase conductivity, ion $/ \mathrm{H}^{+}$transporters), is comprehensively reported. This review focuses on an integrated description of alternative electron transfer pathways and how they contribute to photosynthetic productivity in the context of plant fitness to the environment.

\title{
Introduction
}

The photosynthesis power plant converts the radiant energy of light into electrochemical energy, ultimately used to reduce atmospheric $\mathrm{CO}_{2}$ into $\mathrm{C}_{3}$ intermediates, then $\mathrm{C}_{6}$ compounds, serving as the building blocks for all organic molecules found in plants. These energy rich stocks are much more easily broken down for food or burned off as thermal energy than they are synthesized. We know a lot about the molecular processes of photosynthesis primary energy conversion, involving a series of transmembrane proteinsencompassing light harvesting complexes, photosystems, cytochrome $b_{6} f$ complex and adenosine triphosphate (ATP) synthase. In the last 10-20 years, with the flourishing of molecular genetics, a few additional players have come into play that intriguingly are as important as the primary reactions, because they allow plants to endure fluctuating conditions in the wild. These are often referred to as 'alternative electron transport pathways', and play an indispensable regulatory role for photosynthesis.

Photosynthetic organisms use alternative electron transport to respond rapidly to changes to their environment. The general consensus is that these pathways balance photosynthetic electron transfer so that light energy, converted in the form of ATP and nicotinamide adenine dinucleotide (phosphate) (NADPH), is optimally used for $\mathrm{CO}_{2}$ fixation or safely dissipated as heat. If, for instance, the electron carriers become over-reduced, $\mathrm{O}_{2}$ can replace $\mathrm{CO}_{2}$ as a terminal electron acceptor, creating reactive oxygen species (ROS). Over longer time frames, alternative pathways are a resource to acclimate to a variety of physiological conditions while their activity also limits the potential of photosynthetic yields [1]. They can also play moonlighting roles in non-green tissues, catalyzing essential redox reactions [2]. To be 
comprehensive, we chose to provide information on the whole spectrum of pathways that have been identified, studied and recently reported in the literature but we focus here only on the contribution of alternative pathways to the regulation of the light photosynthetic reactions. Figure 1 provides a 'beginners guide' to these pathways with the most salient points about the proteins involved and how they are currently recognized to function. Our purpose is to show how these pathways interconnect and we describe this as a bioprocess-engineering problem in Figure 2. Recent improvements in whole systems techniques are starting to help explain the redundancy and relatedness of these pathways (see Box 1).

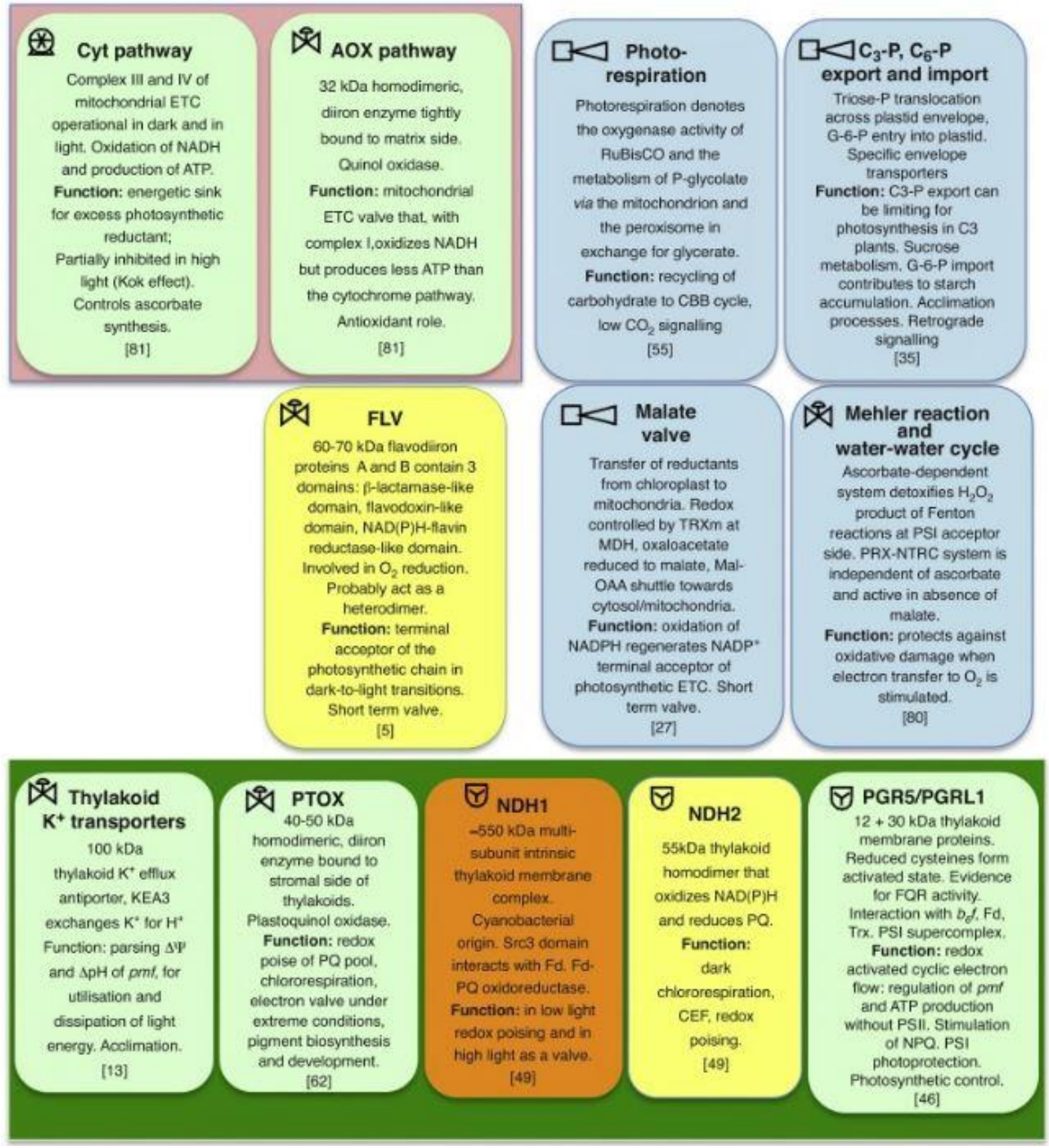

Figure 1. A set of I.D. cards for alternative electron transport pathways organized by their localization (pink background are mitochondrial and dark green in chloroplast thylakoids) describes the structure and functions of a group of the proteins and alternative 
pathways reported in this review and shown schematically in Figure 2. We invite the reader to explore the review(s) suggested for each of these pathways for a more complete overview [5,13,27,35,46,49,55,62,80,81]. Pale green cards are proteins that are conserved in the green lineage; the yellow cards have not been found in angiosperm chloroplasts while the orange card is absent from all algae and the gymnosperms, Gnetales and Pinaceae, sequencedto date. The blue cards show pathways.

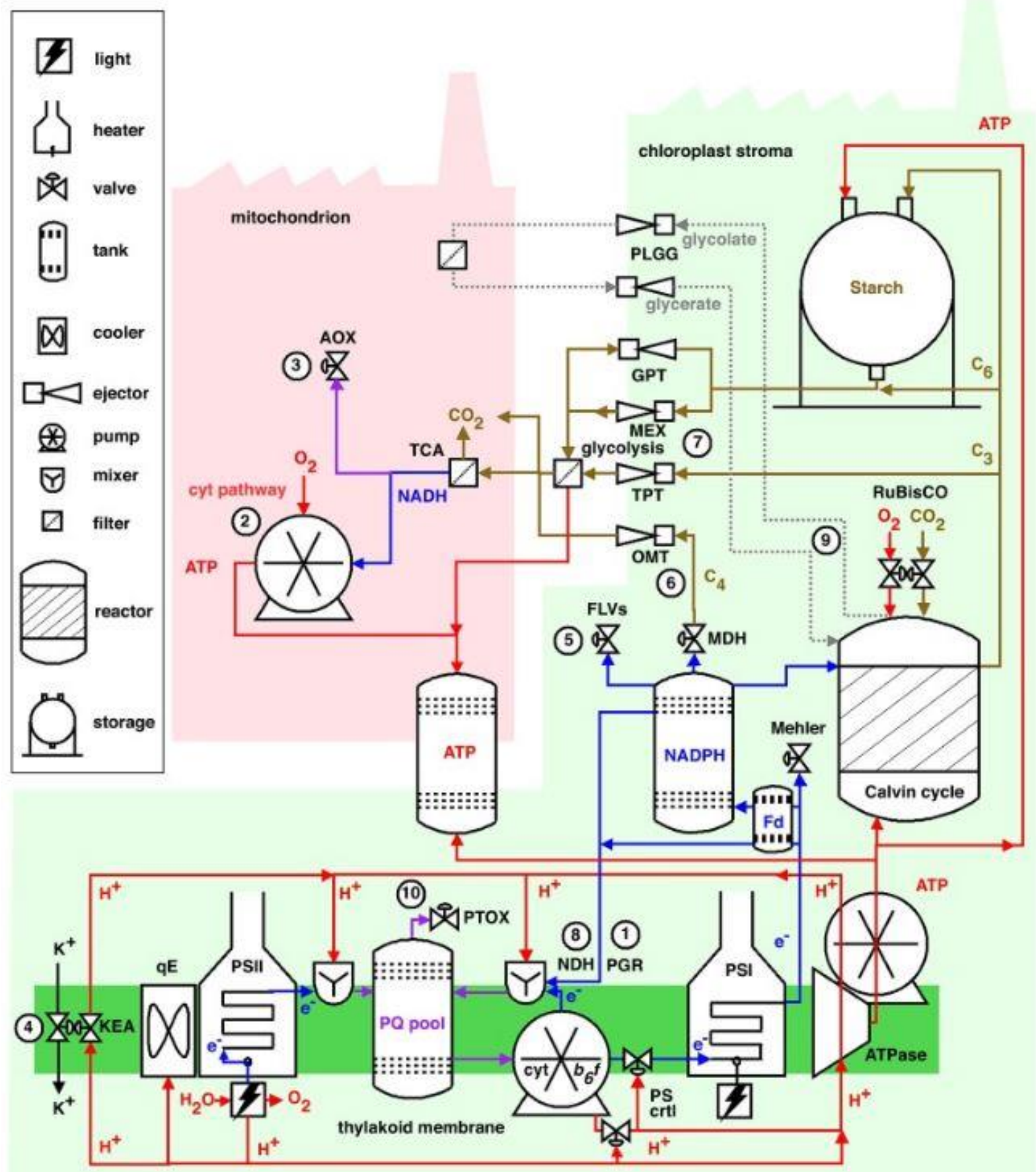

Figure 2. Process flow diagram of the biochemical reactions of photosynthetic eukaryotes. The simplified bioenergetics reactions of respiration are shown in the mitochondrion (pink shading) and the photosynthetic reactions along the thylakoid membrane (green strip) and chloroplast (green shading). Whereas the respiratory chain oxidizes NADH (blue) to 
make ATP (red, oxidative phosphorylation), the photosynthetic chain makes NADPH (blue) and ATP (red). These are used for $\mathrm{CO}_{2}$ capture, synthesis of $\mathrm{C}_{3}$ and $\mathrm{C}_{6}$ metabolites and starch storage (brown). Metabolites are exchanged between various cellular compartments through specific shuttles (shown as 'ejectors'). Alternative pathways are shown as 'valves' (exhaust valves like PTOX, AOX, FLVS or Mehler or flow control valves like PS ctrl); ' $H^{+} / e^{-}$mixers' $\left(P Q+2 H^{+}+2 e^{-} \leftrightarrows P_{2} H_{2}\right)$; or 'coolers' as in dissipation as heat by $N P Q$. They are tagged with numbers to help referencing in the text

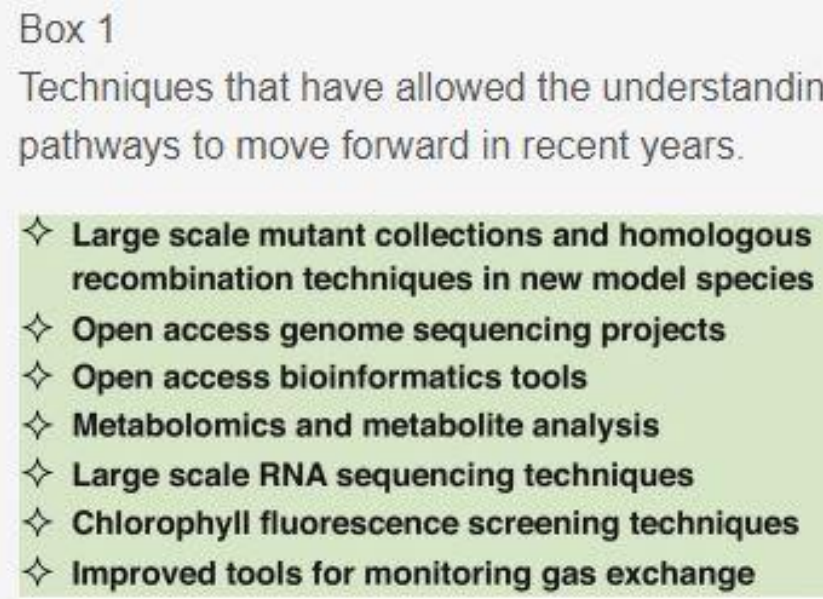

Part I. Redox poising of the photosystem I (PSI) acceptor side is important in fluctuating light and when $\mathrm{CO}_{2}$ availability changes

Cyclic electron flow around PSI, mediated by the PGR5-PGRL1 pathway (see in Figure 2), builds up a proton-motive force across the thylakoid membrane and produces ATP to meet metabolic demands of the chloroplast $[3 *, 4,5]$. It relaxes the reducing pressure at the PSI acceptor side by: (i) providing the Calvin-Benson-Bassham (CBB) cycle with ATP to reoxidize NADPH, (ii) limiting electron transfer upstream of PSI. An increased $\Delta \mathrm{pH}$ component of the proton motive force ( $p m f)$, upregulates non-photochemical quenching $\mathrm{qE}$ and limits the excitation pressure from photosystem II (PSII). $\Delta \mathrm{pH}$ also downregulates linear electron flow at cyt $b_{6} f$ thus the term photosynthetic control (photosynthetic control (PS ctrl) in Figure 2) is used for this valve acting on both electron and proton flow. ATPsynthase conductivity, which is redox controlled and responds to ADP availability and pmf, is also intrinsically linked to this process $[2,6,7]$.

Arabidopsis pgr5 mutants show that PSI is particularly vulnerable to fluctuating light, rather than high light $\left[8^{*}\right]$, due to an imbalanced electron transport chain because of a lack of pmfregulation. In Chlamydomonas, when electrons are in excess due to limiting ATP, $\mathrm{O}_{2}$ photoreduction pathways can take up this load [4,9]. In Arabidopsis, decreasing PSII [10] or introducing a PSI acceptor side sink for electrons [11] can alleviate these effects. In Chlamydomonas [4,9] as well as in Arabidopsis [12] there is evidence that 
the cytochromerespiratory pathway (cyt $b c_{1}$, cyt $c$ and cyt oxidase) rather than alternative pathway(alternative oxidase-AOX) can act as a sink for excess electrons.

The pmf is partitioned between $\Delta \mathrm{pH}$ and $\Delta \psi$. A number of transporters that have specificity for ions, Pi and ADP/ATP have been identified in thylakoid membranes [13]. We concentrate here on the KEA3 thylakoid efflux antiporter because of its apparent specific role in evacuating protons from the lumen (decreasing $\Delta \mathrm{pH}$ ) and importing $\mathrm{K}^{+}$into the lumen (increasing $\Delta \psi$ ) until a steady state is reached [14,15]. The physiological significance of KEA3 has been identified in light transitions to balance $\Delta \mathrm{pH}$ and $\Delta \psi$, an effect that would be attenuated under constant light conditions through downregulation of KEA3 via its luminal C-terminal $[16,17]$. The absence of KEA3 has consequences for the efficiency of photosynthetic electron transport [18], suggesting that partitioning of pmf into $\Delta \mathrm{pH}$ and $\Delta \psi$ components is actively regulated and required for efficient photosynthesis.

Flavodiiron (FLV) proteins $\mathrm{A}$ and $\mathrm{B}$ oxidize NADPH at the expense of $\mathrm{O}_{2}$ and serve as terminal electron acceptors at the onset of light in green algae, bryophytes and gymnosperms [9,19-21]. The characterization of cyanobacterial FLV1/3 proteins as electron acceptors from PSI [22] led to their identification in eukaryotes by sequence homology. Analysis of flva and flvb knock-out mutants in Physcomitrella patens and Marchantia polymorpha, shows that FLV activity contributes to the long-observed transient kinetics of chlorophyll fluorescence, also called Kautsky effect [23], in basal land plants and green algae at the onset of illumination [19,21]. Furthermore FLVs protect PSI integrity in fluctuating light, under these conditions both flva and flvb mutants show severe growth phenotypes [19]. An elegant approach was also used to show that FLVs could function in angiosperms in rapid dark-to-light transitions: the transformation of Atpgr5 plants with Physcomitrella FLVs protected PSI [11]. This study clearly showed that FLVs could act as a valve for electrons at the acceptor side of PSI, stimulating pmf and reinforced the idea that PSI photoinhibition occurs as a consequence of acceptor side limitation [24]. Interestingly however, the $\Delta \mathrm{pH}$-dependent component of NPQ, $\mathrm{qE}$, could only be partially restored in the pgr5 knock-out FLV expressing transgenic lines, reportedly because the parsing of the pmfbetween $\Delta \Psi$ and $\Delta \mathrm{pH}$ was weighted toward $\Delta \Psi$. As already suggested [25] the downregulation of PSII activity via $\mathrm{qE}$ is not the major effector in PSI photoprotection. However, the complementarity of CEF and qE to acceptor side poising in algae may be crucial [26]. The fact that the FLV proteins are a dispensable valve in angiosperms, begs the question of why algae, bryophytes and gymnosperms have retained it.

Malate plays a central role in chloroplast metabolism in the light. It is required for $\mathrm{C} 4$ photosynthesis as the $\mathrm{C} 4$ intermediate that delivers $\mathrm{CO}_{2}$ to ribulose-1,5-bisphosphate carboxylase oxygenase (RuBisCO) via the activity of NADP-malic enzyme in bundle sheath cells. The 'malate valve' , serves a role in C3 photosynthesis whereby chloroplast malate dehydrogenase (NADP-MDH) is activated by reduced thioredoxins and inactivated by NADP ${ }^{+}$. The translocation of malate toward mitochondria via the oxaloacetate/malate transporter (AtpOMT1 in Arabidopsis) provides a short term regulatory response when NADPH production exceeds its consumption in the chloroplast [27]. While the Arabidopsis $m d h$ mutant 
does not show a growth phenotype [28], the Atpomt1 plastid envelope transporter mutant suffers a short-term photoinhibition phenotype when transferred from low light to high light, conditions where the malate valve would be the most important [29]. It is interesting to note that AtpOMT1 also transports 2-oxaloglutarate, a trafficking linked to nitrogen metabolism and photorespiration. A redox activated malate dehydrogenase (MDH) is found in Chlamydomonas and lower plants $[30,31]$ but the assessment of its contribution to the strong interaction between photosynthesis and respiration [4,32] is awaiting specific mutants.

Part II. Alternative pathways act as constitutive valves in low $\mathrm{CO}_{2}$, high light, cold, salt

\section{and drought stress}

Triose-phosphates synthesized from $\mathrm{CO}_{2}$ in the $\mathrm{CBB}$ cycle are directed toward sucrose for export and/or kept in the chloroplast for starch synthesis at a rate that depends on RuBisCOrates and RuBP turnover [33]. At variance with the malate valve that only acts on $\mathrm{NADP}^{+} / \mathrm{NADPH}$ levels, triose-phosphate translocation (TPT) also balances Pi, ADP and ATP. ATPsynthase conductivity and pmf at steady state depends on the availability of these substrates, therefore Triose-P shuttling has consequences on electron and proton transfer [34]. Chloroplast envelopes have large families of TPTs and a number of translocators and mutants have been characterized [35]. In Arabidopsis tpt mutants, an increase in transient pools of starch is witnessed by a futile cycle of starch synthesis and degradation. Mutants can use this transient starch pool as a buffer, but it becomes saturated in high light [36,37]. The rice Ostpt1 mutant is unable to sustain such a compensatory pathway and has a stunted growth phenotype at all light regimes [38]. The balance of phosphorylated sugars inside and outside the chloroplast has a regulatory role in acclimation [39]: arresting either chloroplast export of triose-P in tpt mutants or translocation of glucose-6-phosphate (via GPT2) into the chloroplast in gpt2 mutants and are both associated with a defective acclimation response. This is observed at the transcriptional and metabolic level resulting in an inability to upregulate photosynthetic electron transfer when transferred to high light [36,37,39].

NADPH dehydrogenase (NDH1) is a multi-subunit complex resembling the mitochondrialcomplex I. Compelling evidence shows that despite its name and its evolutionary origin it oxidizes ferredoxin rather than NADPH and uses plastoquinone (PQ) as an electron acceptor [40]. There is evidence that its role would be rather in cyclic electron flow and steady state redox poising in low light in C3 species [41] and its high accumulation and complementarity to proton gradient regulation 5/like 1 (PGR5/PGRL1) is supported in C4 species [42-45]. NDH1 pathway would be minor under high light conditions in C3 plantswhere the PGR5 pathway would be dominant [46]. The type-2 NDH2, a true NADPH dehydrogenase, accumulates in thylakoids of green algae and some gymnosperms in the absence of the type-I $\mathrm{NDH}$. It would appear to have the same function in algae as in $\mathrm{C} 3$ plants in redox poising but also as a valve under anaerobic conditions [47-49]. Under conditions where sustained PSI acceptor-side limitation occurs, increasing evidence suggests that the $p m f$ mostly induced by PGR5-CEF and parsed between $\Delta \Psi$ and $\Delta \mathrm{pH}$ by KEA3, would control PSII photoinhibition [4,10,50-52]. 
Carbon concentrating mechanisms (CCM) are present in both $\mathrm{C} 4$ plants as well as algae via different mechanisms that require extra ATP per $\mathrm{CO}_{2}$ fixed. In Chlamydomonas a clear correlation is found between assimilation of air levels of $\mathrm{CO}_{2}$ and cyclic electron flow [53]. Less defined grana stacks are observed as constitutive traits both in Chlamydomonas and in bundle sheath cells of some $\mathrm{C} 4$ plants favoring increased rates of cyclic electron flow [54]. Interestingly, bundle sheath cells accumulate high levels of both NDH and PGR5 proteins [4245].

In the absence of $\mathrm{CCM}$ or $\mathrm{C} 4$ structure the oxygenase activity of RuBisCO becomes nonnegligible. Photorespiration involves metabolic reactions in the chloroplast, peroxisomeand mitochondrion that represent an integrated network involving carbon and nitrogen metabolism to maintain the CBB cycle active [55]. It can be seen as a photosynthetic valve essential under drought stress [27] that would act at the metabolic level [56] and transcriptional level, with recent data suggesting metabolites produced by photorespiration act in a signaling pathway regulating stomatal opening [57]. At the metabolic level, photorespiration is highly connected to other alternative pathways: (i) under high- $\mathrm{O}_{2}$ and low- $\mathrm{CO}_{2}$ (photorespiratory conditions) mitochondrial activity shows recruitment of the alternative pathway (AOX) rather than the cytochrome pathway [58,59]; (ii) the same signals trigger both photorespiration and the malate valve in continuous high light. Arabidopsis $m d h$ mutants are not light sensitive and this was explained by an upregulation of photorespiration and ROS detoxification [28]; the ATP levels in $m d h$ mutants would be conserved via upregulation of nitrogen metabolism and glycolysis [60]; (iii) under the same conditions when Arabidopsis plants are subjected to low light an interplay occured between photorespiration, Mehler reaction and the malate valve; while under high light, CEF contribution makes up the ATP demand [61].

The chloroplast counterpart of AOX is plastid terminal oxidase (PTOX), bound to the thylakoid membrane, oxidizing $\mathrm{PQH}_{2}$ and reducing $\mathrm{O}_{2}$ to $\mathrm{H}_{2} \mathrm{O}$ [62]. In the dark activity of type$2 \mathrm{NDH}$ to reduce plastoquinone at the expense of NADPH poises the pool by a mechanism known as chlororespiration [62]. In the light, PTOX would serve as a valve for oxidizing the PQ pool, maintaining PSII active and potentially contributing to pmf by $\mathrm{H}^{+}$release in the lumen from water splitting $[63,64]$. To accept electrons from PSII without overproduction of ROS, PTOX would have to be localized within PSII microdomains accessible to quinone diffusion, that is, grana stacks or margins [65,66]. Although, PTOX seemed to be addressed to the lamellae in non-stressed Arabidopsis plants [67], recent studies suggest a control by stromal $\mathrm{pH}$ that may allow PTOX conditional access to plastoquinols [68]. Lodgepole pine uses PTOX and FLV proteins as the major $\mathrm{O}_{2}$ photoreduction pathways during acclimation to cold to generate $\triangle \mathrm{pH}$ for NPQ [69]. PTOX activity can enhance cyclic electron flow in PTOX overexpressor mutants by oxidizing $\mathrm{PQH}_{2}$ thereby providing PQ as an electron acceptor for ferredoxin [65,70]. In cold stressed tropical plants where PSII is photoinhibited, an upregulation of PTOX and PGR5 is observed, providing some physiological relevance to the interplay between these pathways [71].

\section{Conclusions}


The study of photosynthesis identified pathways that were auxiliary to linear electron flow. However, the molecular identification of these pathways came from diverse methods and sometimes occurred in an unexpected manner: ferredoxin-mediated cyclic photophosphorylation (CEF) was identified in isolated spinach thylakoids [72], NDH1 was identified from sequencing of the Tobacco chloroplast genome [73], pgr5 from a chlorophyll fluorescence screen in Arabidopsis [74], PTOX was found in ghost mutants of tomato [75,76]. Whole genome sequencing of members of the green lineage outside the angiosperms identified the FLV proteins by homology with cyanobacterial FLVs [77]. Similar genetic lesions can cause very different phenotypic traits in different organisms, for example, an absence of PTOX was found to result in colorless tomatoes or variegated Arabidopsis leaves [76,78,79], a finding that expanded the alternative pathways reach to developmental processes. The diversity of these observations has enriched our knowledge on the ins and outs of the alternative pathways in a large panel of photosynthetic organisms. Nonetheless, it should not distract us from the similarities that have been shown to exist at the molecular level. The real challenge now is to devise a combination of quantitative and physiological approaches to place each of these pathways back into the integrated network of plant metabolism and to decipher their roles in response to environmental cues.

\section{Acknowledgement}

This work was supported by grants from the Agence Nationale de la

Recherche(ChloroPaths: ANR-14-CE05-0041-01).

\section{References}

1.J. Kromdijk, K. Glowacka, L. Leonelli, S.T. Gabilly, M. Iwai, K.K. Niyogi, S.P. LongImpr oving photosynthesis and crop productivity by accelerating recovery from photoprotection

Science, 354 (2016), pp. 857-861

\section{$2 ;$ K. Kohzuma, C. Dal}

Bosco, A. Kanazawa, A. Dhingra, W. Nitschke, J. Meurer, D.M. KramerThioredoxininsensitive plastid ATP synthase that performs moonlighting functions

Proc. Natl. Acad. Sci. U. S. A., 109 (2012), pp. 3293-3298

3.A.P. Hertle, T. Blunder, T. Wunder, P. Pesaresi, M. Pribil, U. Armbruster, D. LeisterPGRL $\mathbf{1}$ is the elusive ferredoxin-plastoquinone reductase in photosynthetic cyclic electron flow Mol. Cell, 49 (2013), pp. 511-523

The first and latest attempt made to prove that PGR5 and PGRL1 are protein components of a Ferredoxin Quinone Reductase, providing a link between Antimycin A sensitive CEF and these proteins.

4.X. Johnson, J. Steinbeck, R.M. Dent, H. Takahashi, P. Richaud, S. Ozawa, L. HouilleVernes, D.Petroutsos, F. Rappaport, A.R. Grossman, et al.Proton gradient regulation 5mediated cyclic electron flow under ATP- or redox-limited conditions: a study of 
DeltaATpase pgr5 and DeltarbcL pgr5 mutants in the green alga Chlamydomonas reinhardtii

Plant Physiol., 165 (2014), pp. 438-452

5.T. Shikanai, H. YamamotoContribution of cyclic and pseudo-cyclic electron transport to the formation of proton motive force in chloroplasts

Mol. Plant, 9 (Jan (1)) (2016), pp. 20-29

6.K. Kohzuma, C. Dal Bosco, J. Meurer, D.M. KramerLight- and metabolism-related regulation of the chloroplast ATP synthase has distinct mechanisms and functions J. Biol. Chem., 288 (2013), pp. 13156-13163

7.C. Wang, H. Yamamoto, T. ShikanaiRole of cyclic electron transport around photosystem $I$ in regulating proton motive force Biochim. Biophys. Acta, 1847 (2015), pp. 931-938

8.M. Suorsa, S. Jarvi, M. Grieco, M. Nurmi, M. Pietrzykowska, M. Rantala, S. Kangasjarvi, V. Paakkarinen, M. Tikkanen, S. Jansson, et al.PROTON GRADIENT REGULATION5 is essential for proper acclimation of Arabidopsis photosystem I to naturally and artificially fluctuating light conditions

Plant Cell, 24 (2012), pp. 2934-2948

Using pgr5 mutants both in the field and in controlled culture conditions under fluctuating light revealed the function of $\mathrm{CEF}$ in $\mathrm{C} 3$ angiosperms and absence of $\mathrm{CEF}$ was directly linked to damage to PSI proteins in young plants.

9.K.V. Dang, J. Plet, D. Tolleter, M. Jokel, S. Cuine, P. Carrier, P. Auroy, P. Richaud, X. Joh nson, J. Alric, et al.Combined increases in mitochondrial cooperation and oxygen photoreduction compensate for deficiency in cyclic electron flow in Chlamydomonas reinhardtii

Plant Cell, 26 (2014), pp. 3036-3050

10.M. Suorsa, F. Rossi, L. Tadini, M. Labs, M. Colombo, P. Jahns, M.M. Kater, D. Leister, G . Finazzi, E.M.Aro, et al.PGR5-PGRL1-dependent cyclic electron transport modulates linear electron transport rate in Arabidopsis thaliana

Mol. Plant, 9 (2016), pp. 271-288

11.H. Yamamoto, S. Takahashi, M.R. Badger, T. ShikanaiArtificial remodelling of alternative electron flow by flavodiiron proteins in Arabidopsis

Nat. Plants, 2 (2016), p. 16012

By replacing cyclic flows with pseudo cyclic flows, the authors clearly showed that the two pathways have similar functions and are complementary.

12.I. Florez-Sarasa, K. Noguchi, W. Araujo, A. Garcia-

Nogales, A.R. Fernie, J. Flexas, M. Ribas-CarboImpaired cyclic electron flow around photosystem I disturbs high-light respiratory metabolism 
13.B.E. Pfeil, B. Schoefs, C. SpeteaFunction and evolution of channels and transporters in photosynthetic membranes

Cell. Mol. Life Sci., 71 (2014), pp. 979-998

14.U. Armbruster, L.R. Carrillo, K. Venema, L. Pavlovic, E. Schmidtmann, A. Kornfeld, P. Ja hns, J.A. Berry, D.M. Kramer, M.C. JonikasIon antiport accelerates photosynthetic acclimation in fluctuating light environments

Nat. Commun., 5 (2014), p. 5439

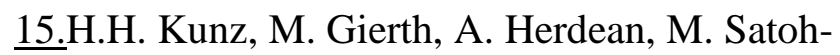

Cruz, D.M. Kramer, C. Spetea, J.I. SchroederPlastidial transporters KEA1, -2, and -3 are essential for chloroplast osmoregulation, integrity, and pH regulation in Arabidopsis Proc. Natl. Acad. Sci. U. S. A., 111 (2014), pp. 7480-7485

16.U. Armbruster, L. Leonelli, V. Correa

Galvis, D. Strand, E.H. Quinn, M.C. Jonikas, K.K. NiyogiRegulation and levels of the thylakoid $\mathrm{K}+/ \mathrm{H}+$ antiporter KEA3 shape the dynamic response of photosynthesis in fluctuating light

Plant Cell Physiol., 57 (Jul (7)) (2016), pp. 1557-1567

A detailed analysis of the different KEA3 isoforms reveals the regulatory domain and function of KEA in optimizing NPQ relaxation and avoiding limitations to CBB in dark light transitions.

17.C. Wang, H. Yamamoto, F. Narumiya, Y.N. Munekage, G. Finazzi, I. Szabo, T. ShikanaiF ine-tuned regulation of the $\mathrm{K}+/ \mathrm{H}+$ antiporter $\mathrm{KEA3}$ is required to optimize photosynthesis during induction

Plant J., 89 (Feb (3)) (2016), pp. 540-553

18.S. Dana, A. Herdean, B. Lundin, C. SpeteaEach of the chloroplast potassium efflux antiporters affects photosynthesis and growth of fully developed Arabidopsis rosettes under short-day photoperiod

Physiol. Plant., 158 (2016), pp. 483-491

19.C. Gerotto, A. Alboresi, A. Meneghesso, M. Jokel, M. Suorsa, E.M. Aro, T. MorosinottoFl avodiiron proteins act as safety valve for electrons in Physcomitrella patens Proc. Natl. Acad. Sci. U. S. A., 113 (2016), pp. 12322-12327

20.P. Ilik, A. Pavlovic, R. Kouril, A. Alboresi, T. Morosinotto, Y. Allahverdiyeva, E.M. Aro, H. Yamamoto, T.ShikanaiAlternative electron transport mediated by flavodiiron proteins is operational in organisms from cyanobacteria up to gymnosperms

New Phytol. (Mar (17)) (2017), 10.1111/nph.14536

21.G. Shimakawa, K. Ishizaki, S. Tsukamoto, M. Tanaka, T. Sejima, C. Miyake 
The liverwort, Marchantia, drives alternative electron flow using a flavodiiron protein to protect PSI

Plant Physiol., 173 (2017), pp. 1636-1647

22.Y. Allahverdiyeva, H. Mustila, M. Ermakova, L. Bersanini, P. Richaud, G. Ajlani, N. Battc hikova, L.Cournac, E.M. Aro

Flavodiiron proteins Flv1 and Flv3 enable cyanobacterial growth and photosynthesis under fluctuating light

Proc. Natl. Acad. Sci. U. S. A., 110 (2013), pp. 4111-4116

23.H. Kautsky, A. Hirsch

Neue Versuche zur Kohlensäureassimilation

Naturwissenschaften, 19 (1931), p. 964

24.F. Chaux, G. Peltier, X. Johnson

A security network in PSI photoprotection: regulation of photosynthetic control, NPQ and $\mathrm{O}_{2}$ photoreduction by cyclic electron flow

Front. Plant Sci., 6 (2015), p. 875

25.M. Tikkanen, S. Rantala, E.M. Aro

Electron flow from PSII to PSI under high light is controlled by PGR5 but not by PSBS Front. Plant Sci., 6 (2015), p. 521

26.F. Chaux, X. Johnson, P. Auroy, A. Beyly-Adriano, I. Te, S. Cuine, G. Peltier

PGRL1 and LHCSR3 compensate for each other in controlling photosynthesis and avoiding photosystem I photoinhibition during high light acclimation of Chlamydomonas cells

Mol. Plant, 9 (Jan (1)) (2017), pp. 216-218

27.Voss, B. Sunil, R. Scheibe, A.S. Raghavendra

Emerging concept for the role of photorespiration as an important part of abiotic stress response

Plant Biol. (Stuttg.), 15 (2013), pp. 713-722

28.I.Hebbelmann, J. Selinski, C. Wehmeyer, T. Goss, I. Voss, P. Mulo, S. Kangasjarvi, E.M. Aro, M.L. Oelze, K.J. Dietz, et al.

Multiple strategies to prevent oxidative stress in Arabidopsis plants lacking the malate valve enzyme NADP-malate dehydrogenase

J. Exp. Bot., 63 (2012), pp. 1445-1459

An exhaustive study of the nadp-mdh mutant in Arabidopsis reveals the extent of remodeling of the mutant's metabolism when the malate shunt is inactive despite the lack of growth phenotype.

29.H. Kinoshita, J. Nagasaki, N. Yoshikawa, A. Yamamoto, S. Takito, M. Kawasaki, T. Sugi yama, H. Miyake, A.P. Weber, M. Taniguchi 
The chloroplastic 2-oxoglutarate/malate transporter has dual function as the malate valve and in carbon/nitrogen metabolism

Plant J., 65 (2011), pp. 15-26

30.S.D. Lemaire, A. Quesada, F. Merchan, J.M. Corral, M.I. Igeno, E. Keryer, E. IssakidisBourguet, M.Hirasawa, D.B. Knaff, M. Miginiac-Maslow

NADP-malate dehydrogenase from unicellular green alga Chlamydomonas reinhardtii. A first step toward redox regulation?

Plant Physiol., 137 (2005), pp. 514-521

31.O. Ocheretina, I. Haferkamp, H. Tellioglu, R. Scheibe

Light-modulated NADP-malate dehydrogenases from mossfern and green algae:

insights into evolution of the enzyme's regulation

Gene, 258 (2000), pp. 147-154

32.G. Forti, A. Furia, P. Bombelli, G. Finazzi

In vivo changes of the oxidation-reduction state of NADP and of the ATP/ADP cellular ratio linked to the photosynthetic activity in Chlamydomonas reinhardtii

Plant Physiol., 132 (2003), pp. 1464-1474

\section{T.D. Sharkey}

Understanding carbon partitioning and its role in determining plant growth

Plant Cell Environ., 38 (2015), pp. 1963-1964

\section{J.T. Yang, A.L. Preiser, Z. Li, S.E. Weise, T.D. Sharkey}

Triose phosphate use limitation of photosynthesis: short-term and long-term effects Planta, 243 (2016), pp. 687-698

35.N. Rolland, G. Curien, G. Finazzi, M. Kuntz, E. Marechal, M. Matringe, S. Ravanel, D. Se igneurin-Berny

The biosynthetic capacities of the plastids and integration between cytoplasmic and chloroplast processes

Annu. Rev. Genet., 46 (2012), pp. 233-264

36.A Schneider, R.E. Hausler, U. Kolukisaoglu, R. Kunze, E. van der

Graaff, R. Schwacke, E. Catoni, M.Desimone, U.I. Flugge

An Arabidopsis thaliana knock-out mutant of the chloroplast triose phosphate/phosphate translocator is severely compromised only when starch synthesis, but not starch mobilisation is abolished

Plant J., 32 (2002), pp. 685-699

37.R.G. Walters, D.G. Ibrahim, P. Horton, N.J. Kruger

A mutant of Arabidopsis lacking the triose-phosphate/phosphate translocator reveals metabolic regulation of starch breakdown in the light

Plant Physiol., 135 (2004), pp. 891-906 
38.S.K. Lee, J.S. Eom, L.M. Voll, C.M. Prasch, Y.I. Park, T.R. Hahn, S.H. Ha, G. An, J.S. Je on

Analysis of a triose phosphate/phosphate translocator-deficient mutant reveals a limited capacity for starch synthesis in rice leaves

Mol. Plant, 7 (2014), pp. 1705-1708

39.M.O. Vogel, M. Moore, K. Konig, P. Pecher, K. Alsharafa, J. Lee, K.J. Dietz

Fast retrograde signaling in response to high light involves metabolite export,

MITOGEN-ACTIVATED PROTEIN KINASE6, and AP2/ERF transcription factors in Arabidopsis

Plant Cell, 26 (2014), pp. 1151-1165

40.H. Yamamoto, L. Peng, Y. Fukao, T. Shikanai

An Src homology 3 domain-like fold protein forms a ferredoxin binding site for the chloroplast NADH dehydrogenase-like complex in Arabidopsis

Plant Cell, 23 (2011), pp. 1480-1493

41.J. Kou, S. Takahashi, D.Y. Fan, M.R. Badger, W.S. Chow

Partially dissecting the steady-state electron fluxes in photosystem I in wild-type and pgr5 and ndh mutants of Arabidopsis

Front. Plant Sci., 6 (2015), p. 758

42.N. Ishikawa, A. Takabayashi, K. Noguchi, Y. Tazoe, H. Yamamoto, S. von

Caemmerer, F. Sato, T. Endo

NDH-mediated cyclic electron flow around photosystem I is crucial for C4

photosynthesis

Plant Cell Physiol., 57 (Oct (10)) (2016), pp. 2020-2028

43.Y.N. Munekage, F. Eymery, D. Rumeau, S. Cuine, M. Oguri, N. Nakamura, A. Yokota, B. Genty, G. Peltier

Elevated expression of PGR5 and NDH-H in bundle sheath chloroplasts in

C4 Flaveria species

Plant Cell Physiol., 51 (2010), pp. 664-668

44.Y.N. Munekage, Y.Y. Taniguchi

Promotion of cyclic electron transport around photosystem I with the development of C4 photosynthesis

Plant Cell Physiol., 57 (2016), pp. 897-903

Provides an interesting hypothesis on how ATP requirements may have driven bundle sheath development and thylakoid structure adjustments to optimize cyclic electron flow in these cells.

45.N. Nakamura, M. Iwano, M. Havaux, A. Yokota, Y.N. Munekage 
Promotion of cyclic electron transport around photosystem I during the evolution of NADP-malic enzyme-type $\mathrm{C} 4$ photosynthesis in the genus Flaveria

New Phytol., 199 (2013), pp. 832-842

46.W. Yamori, T. Shikanai

Physiological functions of cyclic electron transport around photosystem I in sustaining photosynthesis and plant growth

Annu. Rev. Plant Biol., 67 (2016), pp. 81-106

47.T.W. Braukmann, M. Kuzmina, S. Stefanovic

Loss of all plastid ndh genes in Gnetales and conifers: extent and evolutionary significance for the seed plant phylogeny

Curr. Genet., 55 (2009), pp. 323-337

48.C. Desplats, F. Mus, S. Cuine, E. Billon, L. Cournac, G. Peltier

Characterization of Nda2, a plastoquinone-reducing type II NAD(P)H dehydrogenase in chlamydomonas chloroplasts

J. Biol. Chem., 284 (2009), pp. 4148-4157

49.G. Peltier, E.M. Aro, T. Shikanai

NDH-1 and NDH-2 plastoquinone reductases in oxygenic photosynthesis

Annu. Rev. Plant Biol., 67 (2016), pp. 55-80

50.G.A. Davis, A. Kanazawa, M.A. Schottler, K. Kohzuma, J.E. Froehlich, A.W. Rutherford, M. Satoh-Cruz, D. Minhas, S. Tietz, A. Dhingra, et al.

Limitations to photosynthesis by proton motive force-induced photosystem II photodamage

eLife, 5 (2016)

A new mechanism for accumulation of $\Delta \Psi$ as a cause of PSII photoinhibition revealed by the use of a set of gamma ATP synthase mutants and high throughput phenotyping techniques.

51.M. Rott, N.F. Martins, W. Thiele, W. Lein, R. Bock, D.M. Kramer, M.A. Schottler ATP synthase repression in tobacco restricts photosynthetic electron transport, $\mathrm{CO}_{2}$ assimilation, and plant growth by overacidification of the thylakoid lumen Plant Cell, 23 (2011), pp. 304-321

52.M. Tikkanen, N.R. Mekala, E.M. Aro

Photosystem II photoinhibition-repair cycle protects photosystem I from irreversible damage

Biochim. Biophys. Acta, 1837 (2014), pp. 210-215

53.B. Lucker, D.M. Kramer

Regulation of cyclic electron flow in Chlamydomonas reinhardtii under fluctuating carbon availability

Photosynth. Res., 117 (2013), pp. 449-459 
An elegant set of experiments link carbon uptake to CEF in green algae, measured under steady state conditions.

54.L. Dumas, M. Chazaux, G. Peltier, X. Johnson, J. Alric

Cytochrome b6f function and localization, phosphorylation state of thylakoid membrane proteins and consequences on cyclic electron flow

Photosynth. Res., 129 (2016), pp. 307-320

55.M. Hodges, Y. Dellero, O. Keech, M. Betti, A.S. Raghavendra, R. Sage, X.G. Zhu, D.K. A llen, A.P. Weber

Perspectives for a better understanding of the metabolic integration of photorespiration within a complex plant primary metabolism network

J. Exp. Bot., 67 (2016), pp. 3015-3026

56.Florian, Z. Nikoloski, R. Sulpice, S. Timm, W.L. Araujo, T. Tohge, H. Bauwe, A.R. Ferni $\mathrm{e}$

Analysis of short-term metabolic alterations in Arabidopsis following changes in the prevailing environmental conditions

Mol. Plant, 7 (2014), pp. 893-911

57.M. Eisenhut, A. Brautigam, S. Timm, A. Florian, T. Tohge, A.R. Fernie, H. Bauwe, A.P. Weber

Photorespiration is crucial to the dynamic response of photosynthetic metabolism and stomatal movement to altered $\mathrm{CO}_{2}$ availability

Mol. Plant, 9 (Jan(1)) (2017), pp. 47-61

58.C.K. Watanabe, W. Yamori, S. Takahashi, I. Terashima, K. Noguchi

Mitochondrial alternative pathway-associated photoprotection of photosystem II is related to the photorespiratory pathway

Plant Cell Physiol., 57 (2016), pp. 1426-1431

59.Vishwakarma, S.D. Tetali, J. Selinski, R. Scheibe, K. Padmasree

Importance of the alternative oxidase (AOX) pathway in regulating cellular redox and ROS homeostasis to optimize photosynthesis during restriction of the cytochrome oxidase pathway in Arabidopsis thaliana

Ann. Bot., 116 (2015), pp. 555-569

60.J. Selinski, R. Scheibe

Lack of malate valve capacities lead to improved $\mathrm{N}$-assimilation and growth in transgenic $A$. thaliana plants

Plant Signal. Behav., 9 (2014), p. e29057

61.B.J. Walker, D.D. Strand, D.M. Kramer, A.B. Cousins 
The response of cyclic electron flow around photosystem I to changes in photorespiration and nitrate assimilation

Plant Physiol., 165 (2014), pp. 453-462

62.W.J. Nawrocki, N.J. Tourasse, A. Taly, F. Rappaport, F.A. Wollman

The plastid terminal oxidase: its elusive function points to multiple contributions to plastid physiology

Annu. Rev. Plant Biol., 66 (2015), pp. 49-74

63.A.G. Ivanov, D. Rosso, L.V. Savitch, P. Stachula, M. Rosembert, G. Oquist, V. Hurry, N.P . Huner

Implications of alternative electron sinks in increased resistance of PSII and PSI photochemistry to high light stress in cold-acclimated Arabidopsis thaliana Photosynth. Res., 113 (2012), pp. 191-206

64.C. Laureau, R. De Paepe, G. Latouche, M. Moreno-

Chacon, G. Finazzi, M. Kuntz, G. Cornic, P. Streb

Plastid terminal oxidase (PTOX) has the potential to act as a safety valve for excess excitation energy in the alpine plant species Ranunculus glacialis $\mathbf{L}$

Plant Cell Environ., 36 (2013), pp. 1296-1310

65.K. Feilke, P. Streb, G. Cornic, F. Perreau, J. Kruk, A. Krieger-Liszkay

Effect of Chlamydomonas plastid terminal oxidase 1 expressed in tobacco on photosynthetic electron transfer

Plant J., 85 (2016), pp. 219-228

66.G.N. Johnson, P. Stepien

Plastid terminal oxidase as a route to improving plant stress tolerance: known knowns and known unknowns

Plant Cell Physiol., 57 (2016), pp. 1387-1396

67.A.M. Lennon, P. Prommeenate, P.J. Nixon

Location, expression and orientation of the putative chlororespiratory enzymes, Ndh and IMMUTANS, in higher-plant plastids

Planta, 218 (2003), pp. 254-260

68.Krieger-Liszkay, K. Feilke

The dual role of the plastid terminal oxidase PTOX: between a protective and a prooxidant function

Front. Plant Sci., 6 (2015), p. 1147

69.L.V. Savitch, A.G. Ivanov, M. Krol, D.P. Sprott, G. Oquist, N.P. Huner

Regulation of energy partitioning and alternative electron transport pathways during cold acclimation of lodgepole pine is oxygen dependent

Plant Cell Physiol., 51 (2010), pp. 1555-1570 
70.Y. Okegawa, Y. Kobayashi, T. Shikanai

Physiological links among alternative electron transport pathways that reduce and oxidize plastoquinone in Arabidopsis

Plant J., 63 (2010), pp. 458-468

71.M.V. Segura, M.J. Quiles

Involvement of chlororespiration in chilling stress in the tropical species Spathiphyllum wallisii

Plant Cell Environ., 38 (2015), pp. 525-533

\section{K. Tagawa, H.Y. Tsujimoto, D.I. Arnon}

Role of chloroplast ferredoxin in the energy conversion process of photosynthesis Proc. Natl. Acad. Sci. U. S. A., 49 (1963), pp. 567-572

73.K. Shinozaki, M. Ohme, M. Tanaka, T. Wakasugi, N. Hayashida, T. Matsubayashi, N. Zait a, J.Chunwongse, J. Obokata, K. Yamaguchi-Shinozaki, et al.

The complete nucleotide sequence of the tobacco chloroplast genome: its gene organization and expression

EMBO J., 5 (1986), pp. 2043-2049

74.Y. Munekage, M. Hojo, J. Meurer, T. Endo, M. Tasaka, T. Shikanai

PGR5 is involved in cyclic electron flow around photosystem $I$ and is essential for photoprotection in Arabidopsis

Cell, 110 (2002), pp. 361-371

75.P.A. Scolnik, P. Hinton, I.M. Greenblatt, G. Giuliano, M.R. Delanoy, D.L. Spector, D. Poll ock

Somatic instability of carotenoid biosynthesis in the tomato ghost mutant and its effect on plastid development

Planta, 171 (1987), pp. 11-18

76.E.M. Josse, A.J. Simkin, J. Gaffe, A.M. Laboure, M. Kuntz, P. Carol

A plastid terminal oxidase associated with carotenoid desaturation during chromoplast differentiation

Plant Physiol., 123 (2000), pp. 1427-1436

77.G. Peltier, D. Tolleter, E. Billon, L. Cournac

Auxiliary electron transport pathways in chloroplasts of microalgae

Photosynth. Res., 106 (2010), pp. 19-31

78.P. Carol, D. Stevenson, C. Bisanz, J. Breitenbach, G. Sandmann, R. Mache, G. Coupland, M. Kuntz

Mutations in the Arabidopsis gene IMMUTANS cause a variegated phenotype by inactivating a chloroplast terminal oxidase associated with phytoene desaturation Plant Cell, 11 (1999), pp. 57-68 
79.D. Wu, D.A. Wright, C. Wetzel, D.F. Voytas, S. Rodermel

The IMMUTANS variegation locus of Arabidopsis defines a mitochondrial alternative oxidase homolog that functions during early chloroplast biogenesis

Plant Cell, 11 (1999), pp. 43-55

80.G. Curien, S. Flori, V. Villanova, L. Magneschi, C. Giustini, G. Forti, M. Matringe, D. Pet routsos, M. Kuntz, G. Finazzi

The water to water cycles in microalgae

Plant Cell Physiol., 57 (2016), pp. 1354-1363

81.P. Schertl, H.-P. Braun

Respiratory electron transfer pathways in plant mitochondria

Front. Plant Sci., 5 (2014), p. 163 